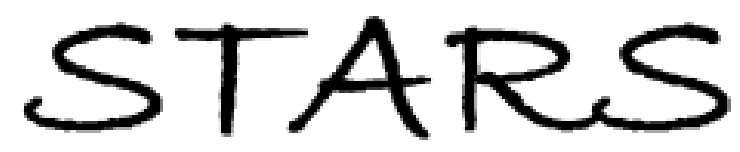

University of Central Florida

STARS

$1-1-2009$

\title{
Analysis and design of wide-angle foveated optical systems based on transmissive liquid crystal spatial light modulators
}

George Curatu

James E. Harvey

University of Central Florida

Find similar works at: https://stars.library.ucf.edu/facultybib2000

University of Central Florida Libraries http://library.ucf.edu

This Article is brought to you for free and open access by the Faculty Bibliography at STARS. It has been accepted for inclusion in Faculty Bibliography 2000 s by an authorized administrator of STARS. For more information, please contact STARS@ucf.edu.

\section{Recommended Citation}

Curatu, George and Harvey, James E., "Analysis and design of wide-angle foveated optical systems based on transmissive liquid crystal spatial light modulators" (2009). Faculty Bibliography 2000s. 1447.

https://stars.library.ucf.edu/facultybib2000/1447

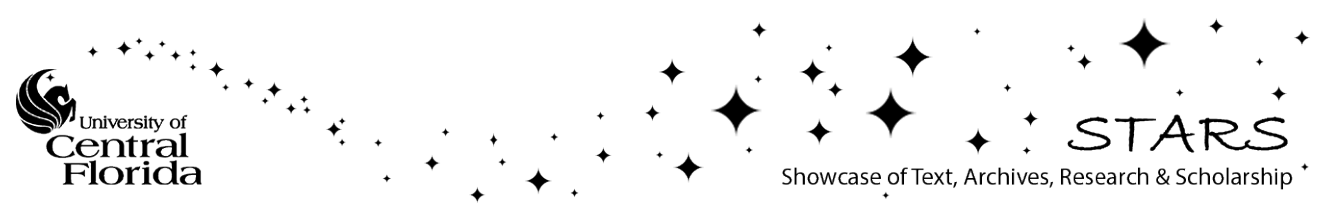




\section{Analysis and design of wide-angle foveated optical systems based on transmissive liquid crystal spatial light modulators}

\author{
George Curatu, MEMBER SPIE \\ LightPath Technologies, Inc. \\ 2603 Challenger Tech Court \\ Orlando, Florida 32826 \\ E-mail: gcuratu@ lightpath.com
}

\author{
James E. Harvey, MEMBER SPIE \\ University of Central Florida \\ CREOL, The College of Optics and Photonics \\ P.O. Box 162700 \\ Orlando, Florida 32816 \\ E-mail: harvey@creol.ucf.edu
}

\begin{abstract}
Optical foveated imaging using liquid crystal (LC) spatial light modulators (SLMs) has received considerable attention in recent years as a potential approach to reducing size and complexity in fast wideangle lenses. We cover a theoretical study quantifying the diffraction efficiency and image quality of foveated optical systems (FOSs) based on transmissive LC SLMs. A practical design example of a fast wideangle FOS based on the current transmissive LC SLM technology is proposed. () 2009 Society of Photo-Optical Instrumentation Engineers. [DOI: 10.1117/1.3122006]
\end{abstract}

Subject terms: foveated imaging; aberration correction; adaptive optics; wideangle lenses; liquid crystal spatial light modulators (SLMs).

Paper 080901R received Nov. 17, 2008; revised manuscript received Feb. 10, 2009; accepted for publication Feb. 26, 2009; published online Apr. 27, 2009.

\section{Introduction}

The development of compact imaging systems capable of transmitting high-resolution images in real time while covering a wide field of view (FOV) is critical in a variety of military and civilian applications: surveillance, threat detection, target acquisition, tracking, remote operation of unmanned vehicles, etc. Foveated imaging was proposed as a data compression technique to speed up transmission and processing of high-resolution digital video frames by reducing the resolution of the image with the exception of a region of interest (ROI), which can be dynamically repositioned anywhere within the FOV. ${ }^{1,2}$ This multiresolution video compression technique was inspired by the operation of the human vision apparatus and is ideal for navigation, surveillance, tracking, display, and other applications where images from a wide FOV have to be transmitted and processed in real time yet high resolution is required at the ROI. Foveated imaging can be achieved at the software level, by applying foveation algorithms to full-resolution digital video frames from a conventional imaging system, ${ }^{1-3}$ or it can be achieved at the hardware level, by combining images from several sensors ${ }^{4,5}$ or by using sensor arrays with variable resolution. ${ }^{6-8}$

Reducing the size and complexity of the optical system is another important task in foveated imaging applications requiring fast (low $\mathrm{F} / \#$ ) and lightweight wide-angle optics. Such lenses are often used in surveillance, navigation of unmanned vehicles, tracking, threat detection, and other applications where a large FOV has to be covered constantly, in different lighting conditions. The large aperture (low $\mathrm{F} / \#)$ is generally needed to gather more light onto the sensor in outdoor applications, where poor ambient lighting can result in a low SNR and therefore, poor detection capabilities. On the other hand, aberrations in the lens increase quickly with the aperture and the field angle, due to

0091-3286/2009/\$25.00 @ 2009 SPIE the severe "ray bending." As a result, fast wide-angle lenses typically require complex designs with multiple elements, in order to carefully balance and correct these aberrations. ${ }^{9}$

Martinez et al. proposed a compact wide-angle lens with variable resolution across the FOV to reduce the size and complexity of wide-angle optics in foveated imaging systems. ${ }^{10}$ The fundamental concept behind the optical foveated imaging technique described by Martinez et al. is reducing the number of elements in a fast wide-angle lens by placing a transmissive phase spatial light modulator (SLM) at the pupil stop to dynamically compensate aberrations left uncorrected by the optical design at preselected points within the FOV. Such a hybrid lens would form an aberrated image over its wide FOV with the exception of a highly resolved ROI, which could be dynamically positioned anywhere within the FOV by adjusting the optical path difference (OPD) pattern introduced by the SLM to correct the wavefront aberration at the desired field angle. A great advantage of the proposed foveated optical system (FOS) is that it could be combined with variable resolution sensors in order to develop compact high-resolution wideangle foveated imaging systems for applications where lightweight, fast data transmission, and low power consumption are critical requirements.

\section{Background}

The phase SLM is the key component enabling optical foveated imaging. Phase SLMs are reflective or transmissive devices used to control the optical wavefront by dynamically changing the OPD across the aperture. Two different types of phase SLMs based on two different technologies have emerged as the most commonly used devices in applications requiring wavefront correction: deformable mirrors (DM), as reflective devices; and liquid crystal (LC) phase SLMs, which can be reflective or transmissive. Reflective devices such as DMs and reflective LC SLMs have been commercially available for many years and have several advantages over transmissive LC SLMs, which seem to 
be the only practical transmissive devices currently available. For instance, segmented DMs have very good zeroorder diffraction efficiencies, large phase strokes, and no dispersion, allowing multispectral applications, and each element can have piston-tip-tilt correction capabilities to minimize the residual wavefront error (RWFE). ${ }^{11}$ Reflective LC SLMs also offer some advantages over transmissive LC SLMs, since they have larger pixel fill factors and smooth transitions between pixels, maximizing the zeroorder diffraction efficiency. ${ }^{12}$

Both DMs and reflective LC SLMs have been used to reduce complexity and improve performance in telescope FOSs. ${ }^{13-15}$ Since the FOV of telescopes is relatively small, these optical systems can be easily folded, allowing the possibility of placing a reflective SLM device at the pupil stop, without blocking the FOV. However, the optical design arrangements in wide-angle systems are fundamentally different than in the case of telescopes, posing particular design challenges related to placing a reflective SLM at the stop. Although a few research groups have attempted to demonstrate wide-angle FOSs using reflective SLM devices, these systems were not practical, ending up bulky, slow (large F/\#), or covering a limited FOV. ${ }^{16-18}$ The problem with using reflective devices in wide-angle FOSs is the fold in the optical axis required after the reflection at the aperture stop. Although achievable in slower systems with narrower FOVs, such folded designs are not practical in the case of fast wide-angle lenses, which typically use a retrofocus configuration, with the stop positioned internally, between a front negative group and a back positive group. A rather large beamsplitting optics would be needed to capture all the rays emerging from the stop and fold the optical axis, sending the rays through the positive group into the image plane. In the case of practical fast wide-angle lenses, the position of the stop with respect to the adjacent optical elements does not allow enough space to insert such a large beamsplitter.

Currently, transmissive LC SLMs seem to be the only transmissive devices available that could potentially allow the development of practical fast wide-angle FOSs. However, there are several fundamental limitations intrinsic to the current transmissive SLM technology. High-resolution transmissive SLMs are based on the same thin-filmtransistor (TFT) technology used in transmissive liquid crystal displays (LCD). A drawback of this technology is that when used in transmissive devices, the active area of each pixel is limited by a shadow mask, which is placed over the transistors and the wiring electronics to prevent photoconduction, as shown in Fig. 1(a). The shadow mask reduces the SLM pixel fill factor, affecting the diffraction efficiency and the image quality of the FOS. The fill factor problem can be somewhat alleviated in transmissive LCDs by using a microlens array layer over the cell to concentrate light through the active pixel area in order to increase illumination. However, using microlens arrays in the case of transmissive SLMs is not practical, since SLMs are used to control the phase rather than displaying illuminated amplitude patterns, as in the case of the LCDs. Decreasing the size of the electronics could be a solution to increasing the fill factor in transmissive SLMs, but there are technological limitations to how much further the electronics and shadow mask can be shrunk. For instance, Sony recently introduced

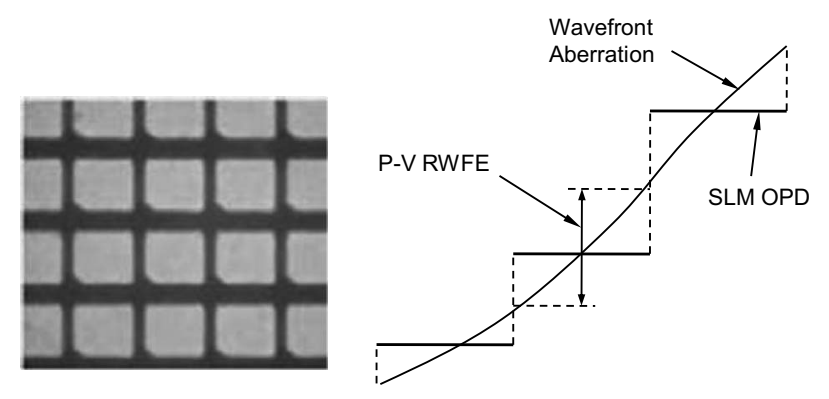

(a)

(b)

Fig. 1 Limitations intrinsic to the transmissive LC SLM technology affect the diffraction efficiency and image quality of FOSs: (a) the fill factor, due to the shadow mask; and (b) the RWFE, due to the discrete piston-only correction.

BrightEra, a new line of state-of-the-art, high-resolution transmissive LCDs based on TFT technology, with the shadow mask reduced from $3.8 \mu \mathrm{m}$ to $2.8 \mu \mathrm{m}$, which seems to be the current minimum mask width in transmissive TFT devices.

Another limitation imposed by the transmissive LC SLM technology is the discrete OPD pattern introduced by the SLM, with piston-only correction at each element, which generates a periodic residual wavefront error (RWFE) at every pixel, as illustrated in Fig. 1(b). The RWFE produces a quasi-periodic phase structure that further affects the diffraction efficiency and the image quality of the FOS.

The amount of signal onto the sensor and the image quality are the two most important performance characteristics of an optical imaging system. In the case of FOSs based on current transmissive LC SLMs, diffraction is the main factor affecting both of these characteristics at the ROI. A significant diffractive effect is caused by the periodic amplitude gridlike structure created by the shadow mask, which is equivalent to the effect of a 2-D amplitude grating. Another diffractive effect is caused by the RWFE, which forms a periodic sawtoothlike phase structure with the same period as the amplitude grating, which has the effect of a 2-D blazed grating with the blaze angle slowly varying across the pupil. The amplitude and phase diffraction caused by the pixelated structure of the transmissive LC SLMs affects the transmission, the zero-order diffraction efficiency, and the image quality of the FOS.

In recent years, considerable research and development has been conducted in the area of optical foveated imaging using the LC SLM technology, and several FOSs have been built. ${ }^{16,17,19-22}$ However, most research has been focused so far on the experimental demonstration of the basic principle using off-the-shelf components, without much concern for the practicality or the optical performance of the systems.

Published results quantify only the aberration correction capabilities of the FOS, often claiming diffraction-limited performance at the ROI, yet constantly overlooking the effects of diffraction on the zero-order efficiency and the image quality. Understanding and quantifying the diffraction effects caused by the current transmissive LC SLMs could help develop practical, fast wide-angle FOSs based on the 


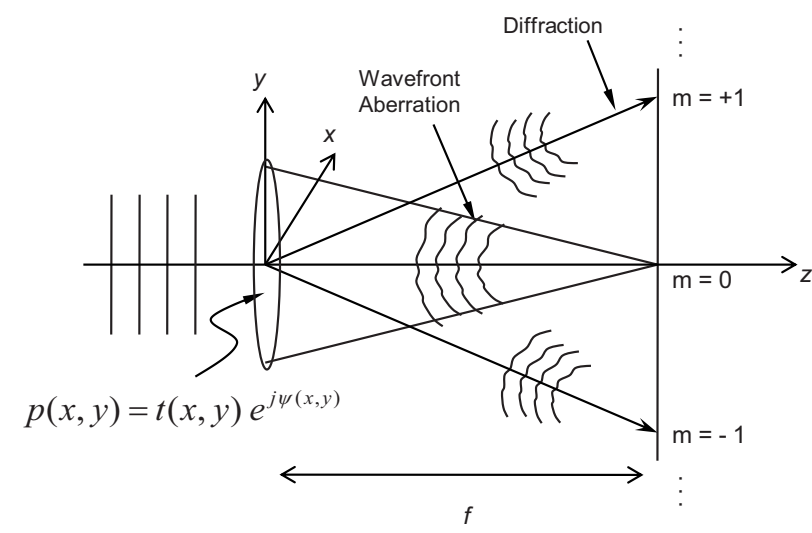

Fig. 2 Diffraction and aberrations are determined by the pupil function.

current technology and also set target requirements and specifications for future developments in the area of transmissive SLMs.

This paper covers a theoretical study quantifying the optical performance of FOSs based on transmissive LC SLMs in terms of their zero-order diffraction efficiency and modulation transfer function (MTF). Diffraction phenomena occurring due to the pixelated SLM aperture are analyzed, estimating their effect on the diffraction efficiency and the MTF. A practical design example of a fast wideangle FOS is presented. The analysis covered in this paper is limited to monochromatic systems, but it can be expanded to polychromatic systems, by considering a wavelength-dependent RWFE over the entire wavelength range.

\section{The Pupil Function}

Considering the rectangular coordinate system defined in Fig. 2, the complex pupil function of an optical system can be written as

$p(x, y)=t(x, y) \exp [j \psi(x, y)]$,

where $t(x, y)$ is the pupil amplitude transmission function, and $\psi(x, y)$ is the phase transmission function. In order to quantify the effects of the pixelated aperture on the performance of an FOS based on transmissive LC SLMs, we have to describe mathematically the typical pupil amplitude and phase functions in these systems.

We start first with the amplitude component of the pupil, $t(x, y)$. Figure 3 illustrates the typical geometry of the amplitude transmission in a transmissive LC SLM. The dark area represents the electronics shadow mask and the obscuration due to the circular aperture of the optics. Consider the following notation: $a$ is the SLM pixel pitch, $b$ is the active pixel width, and $D$ is the diameter of the aperture (assuming a circular aperture). The fill factor is defined as the ratio between the transparent area and the total area of the pixel, so in our case, the fill factor is $(b / a)^{2}$. We can write the amplitude transmission function of the pupil as a 2-D train of rectangle functions of width $b$, spaced apart by the pixel pitch $a$ on $x$ and $y$, and delimited by the circular aperture of diameter $D$ :

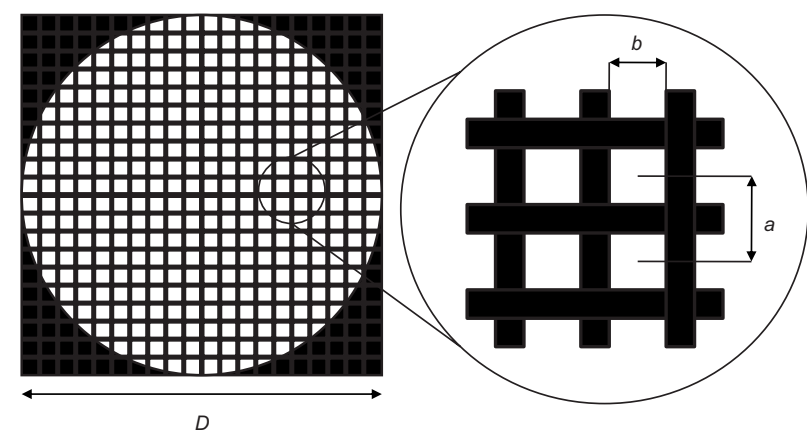

Fig. 3 Amplitude transmission of the pupil of an FOS with transmissive LC SLM- $a$ is the SLM pixel pitch, $b$ is the active pixel width, and $D$ is the diameter of the aperture.

$$
\begin{aligned}
t(x, y)= & {\left[\operatorname{rect}\left(\frac{x}{b}\right) * \frac{1}{a} \operatorname{comb}\left(\frac{x}{a}\right)\right] } \\
& \times\left[\operatorname{rect}\left(\frac{y}{b}\right) * \frac{1}{a} \operatorname{comb}\left(\frac{y}{a}\right)\right] \operatorname{circ}\left[\frac{\left(x^{2}+y^{2}\right)^{1 / 2}}{D / 2}\right] .
\end{aligned}
$$

Now, we look at the phase component, $\psi(x, y)$. In the case of an FOS, the wavefront aberration at the ROI, $W(x, y)$, is corrected by the SLM, with the exception of the RWFE, which is the uncorrected aberration left at each pixel after the SLM correction is applied, as illustrated in Fig. 1(b). The RWFE is a consequence of the discrete piston-only OPD of the transmissive LC SLM. For a large number of pixels $(N \times N, N>32)$, the local RWFE at each pixel can be approximated by a wavefront tilt on $x$ and $y$, as shown in Fig. 4. The local P-V RWFE on $x$ and $y$ at the pixel $[i, j]$ is given by the local slope of the wavefront aberration and the pixel pitch of the SLM:

$$
\begin{aligned}
& R W F E_{P-V x}[i, j]=a \times\left|\frac{\partial W(x, y)}{\partial x}\right|_{x=x_{i}, y=y_{j}}, \\
& R W F E_{P-V y}[i, j]=a \times\left|\frac{\partial W(x, y)}{\partial y}\right|_{x=x_{i}, y=y_{j}},
\end{aligned}
$$

where $x_{i}$ and $y_{i}$ are the pupil coordinates at the pixel $[i, j]$.

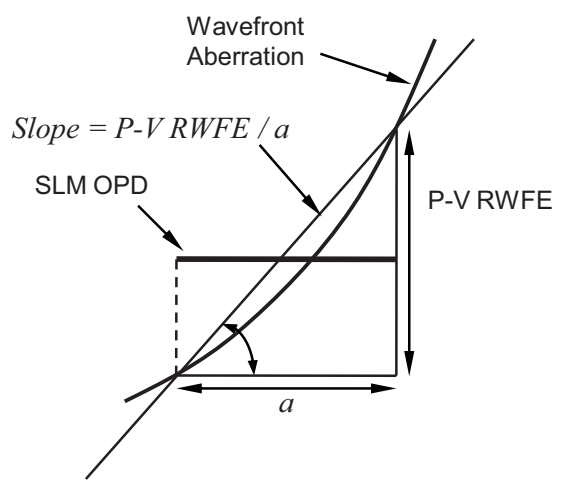

Fig. 4 The local RWFE at each pixel can be approximated by a wavefront tilt. 


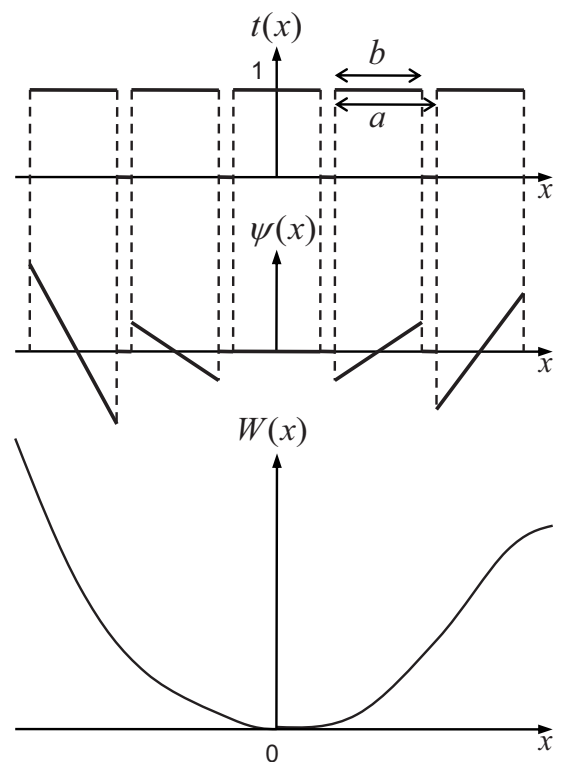

Fig. 5 One-dimensional pupil amplitude and phase transmission plots, $t(x)$ and $\psi(x)$, after correcting the aberration $W(x)$ (period exaggerated for clarity).

After the wavefront aberration is corrected by the SLM, the transmitted pupil phase at the ROI will have the shape of a 2-D periodic sawtooth function, which has the effect of a blazed transmission phase grating, with the period $a$, and the blaze angle given by the slope of the wavefront aberration. The blaze angle will follow a similar variation across the pupil as the slope of the wavefront aberration. The transmitted phase of an FOS with an SLM resolution of $N \times N$ at the wavelength $\lambda$ can be written as

$$
\begin{aligned}
\psi(x, y)= & \frac{2 \pi}{\lambda} \sum_{i, j=1}^{N}\left[\left.\frac{\partial W(x, y)}{\partial x}\right|_{\substack{x=x_{i} \\
y=y_{j}}} x\right. \\
& \left.+\left.\frac{\partial W(x, y)}{\partial y}\right|_{\substack{x=x_{i} \\
y=y_{j}}} y\right] t(x, y) .
\end{aligned}
$$

Figure 5 shows 1-D schematic plots of the pupil amplitude and phase of an FOS based on transmissive LC SLMs after correcting the wavefront aberration at the ROI. The amplitude, $t(x)$, has a value of one at the regions where the SLM is transparent and zero where the SLM is opaque. The phase, $\psi(x)$, looks like a blazed grating with the blaze angle following the variation of the slope of the corrected wavefront aberration, $W(x)$.

\section{Diffraction Efficiency}

The amount of signal onto the sensor in an optical system depends on the aperture ratio $(\mathrm{F} / \#)$, transmission, and diffraction efficiency of the system. In FOSs based on transmissive LC SLMs, a significant loss in signal is caused by the zero-order diffraction efficiency. The periodic structure of the pupil amplitude and phase produces a grating effect, creating higher diffraction orders and limiting the zeroorder efficiency. This section covers a derivation of the diffraction efficiency of an FOS with an SLM resolution of
$N \times N$ as a function of $a, b$, and the P-V RWFE on $x$ and $y$ at every pixel $[i, j]$, as defined in Eq. (3). Note that the symbols $D, a$, and $b$ refer to the physical dimensions in the stop scaled to the size of the entrance pupil. So if the entrance pupil of the optical system is not the same as the stop, the physical dimensions have to be scaled by the magnification of the stop in the object space, which is given by the ratio between the entrance pupil diameter and the stop diameter.

The efficiency of each diffraction order can be calculated from the power spectral density in the image plane, which is the magnitude squared of the field. The field in the image plane is given by the Fourier transform of the field in the pupil. ${ }^{23}$ As a result, the diffraction efficiency can be calculated starting from the complex pupil function, $p(x, y)$, which completely describes the amplitude and phase of the field in the pupil. In order to simplify calculations, we can start by considering a few reasonable assumptions and then generalize the end results. If $D \gg a$, we can consider the case of an infinite aperture, which eliminates the term $\operatorname{circ}\left[\left(x^{2}+y^{2}\right)^{1 / 2} / D / 2\right]$ in Eq. (2). Also, we can only derive the 1-D diffraction efficiency and then extrapolate the result for a 2-D pupil. Furthermore, we can consider the particular case where the wavefront aberration to be corrected at the ROI is only a tilt on $x$ with the slope equal to $R W F E_{P-V} / a$. In this particular case, after the wavefront aberration at the ROI is corrected by the SLM, the pupil becomes a transmission phase grating with a constant blaze angle, so the 1-D pupil function on $x$ can be written as

$p(x)=\left[\operatorname{rect}\left(\frac{x}{b}\right) * \frac{1}{a} \operatorname{comb}\left(\frac{x}{a}\right)\right] \exp \left(j \frac{2 \pi}{\lambda} \frac{R W F E_{P-V}}{a} x\right)$.

Assuming monochromatic plane waves coming from a point source at infinity, the field in the image plane is given by the Fourier transform of the pupil, $p(x)$ :

$P(\xi)=\frac{b}{a} \operatorname{sinc}\left[b\left(\xi-\frac{R W F E_{P-V}}{\lambda a}\right)\right] a \operatorname{comb}(a \xi)$,

where $\xi=x / \lambda f$ is the spatial frequency, $\lambda$ is the wavelength of the incident light, and $f$ is the focal length of the optical system. The power spectral density in the image plane is given by the magnitude squared of the field:

$|P(\xi)|^{2}=\left(\frac{b}{a}\right)^{2} \operatorname{sinc}^{2}\left[b\left(\xi-\frac{R W F E_{P-V}}{\lambda a}\right)\right] a^{2} \operatorname{comb}^{2}(a \xi)$.

The 1-D power spectral density in the image plane is schematically plotted in Fig. 6, in the case where $b>a / 2$ and $R W F E_{P-V}<\lambda$. The diffraction angles are determined by the locations of the delta functions:

$\beta_{m}=\sin ^{-1}\left(\sin \theta+\frac{m \lambda}{a}\right)$,

where $\beta_{m}$ is the diffraction angle of the $m$-order with respect to the optical axis in object space, and $\theta$ is the field angle in object space. The efficiency of each diffraction order is given by the height of the delta function at the corresponding spatial frequency. From Eq. (7), the $m$-order 


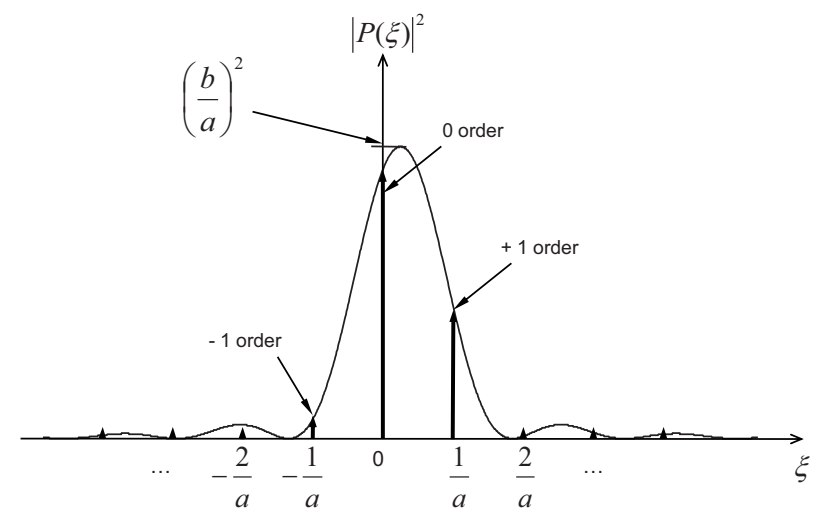

Fig. 6 Power spectral density of a 1-D blazed grating.

diffraction efficiency relative to the total incident light is given by

$$
\sigma_{m}=\left|P\left(\xi=\frac{m}{a}\right)\right|^{2}=\left(\frac{b}{a}\right)^{2} \operatorname{sinc}^{2}\left[\frac{b}{a}\left(m-\frac{R W F E_{P-V}}{\lambda}\right)\right] .
$$

Notice that, for a given grating period, $a$, the blaze angle (RWFE) has the effect only of shifting the distribution of the efficiency among diffracted orders and does not affect the diffraction angles or the total transmission. For instance, if $R W F E_{P-V}=0$, the highest diffraction efficiency will be in the zero-order. If $R W F E_{P-V}=\lambda$, the highest efficiency will be in the +1 -order.

So the diffraction angles in an FOS depend on the SLM pixel pitch, and the total transmission and the diffraction efficiency in each order depend on the pixel fill factor and the RWFE. However, the RWFE in an FOS varies across the pupil on $x$ and $y$, depending on the shape of the wavefront aberration and the OPD introduced by the SLM. Therefore, in order to calculate the diffraction efficiency for any wavefront aberration, the result in Eq. (9) has to be generalized for a 2-D pupil with variable RWFE across $x$ and $y$. Assuming an FOS with a high-resolution SLM, the RWFE varies relatively slowly over several pixels, so for each order, $\left(m_{x}, m_{y}\right)$, we can define the local 2-D diffraction efficiency at every pixel $[i, j]$ as the product between the local diffraction efficiencies on $x$ and $y$ :

$$
\begin{aligned}
\sigma_{m_{x}, m_{y}}[i, j]= & \left(\frac{b}{a}\right)^{4} \operatorname{sinc}^{2}\left[\frac{b}{a}\left(m_{x}-\frac{R W F E_{P-V x}[i, j]}{\lambda}\right)\right] \\
& \times \operatorname{sinc}^{2}\left[\frac{b}{a}\left(m_{y}-\frac{R W F E_{P-V y}[i, j]}{\lambda}\right)\right],
\end{aligned}
$$

where $m_{x}$ and $m_{y}$ are the diffraction orders on $x$ and on $y$, and $R W F E_{P-V x}[i, j]$ and $R W F E_{P-V y}[i, j]$ are the P-V RWFE values on $x$ and on $y$ at the pixel $[i, j]$, as defined in Eq. (3). The term $(b / a)^{4}$ in Eq. (10) represents the diffraction efficiency due to the amplitude part of the pupil function (the electronics shadow mask), and the sinc ${ }^{2}$ terms represent the local diffraction efficiency on $x$ and $y$ caused by the phase part of the pupil function (the RWFE). The term $b / a$ in the argument of the two sinc ${ }^{2}$ functions represents the truncation factor of the local P-V RWFE due to the limited active pixel width. Assuming an SLM resolution of $N \times N$, the overall diffraction efficiency for any order can be calculated as the root-mean-square (RMS) across the entire pupil:

$\sigma_{m_{x}, m_{y}}=\left[\frac{\sum_{i, j=1}^{N}\left(\sigma_{m_{x}, m_{y}}[i, j]\right)^{2}}{N^{2}}\right]^{1 / 2}$.

The total transmission of all diffraction orders combined is equal to the fill factor:

$\sigma_{\text {total }}=\left(\frac{b}{a}\right)^{2}$.

Note that when calculating the diffraction efficiency for each order in Eq. (10), the total transmission is already included in the term $(b / a)^{4}$.

The distribution of the diffraction efficiency among higher orders at the ROI depends on the shape and symmetry of the wavefront aberration corrected at the ROI. For instance, if the wavefront aberration is rotationally symmetric about the optical axis, such as the aberration of an optical system on-axis, the diffraction efficiency will be distributed evenly among the symmetrical orders about the origin and about $x$ and $y$ axes $\left(\sigma_{ \pm m, \pm m}=\right.$ constant and $\left.\sigma_{ \pm m, 0}=\sigma_{0, \pm m}\right)$. In the case of nonsymmetrical aberrations, the diffraction efficiency is distributed according to the overall slope of the wavefront aberration across the pupil.

\section{Modulation Transfer Function}

The image quality of an optical system is affected mainly by three factors: diffraction, aberrations not corrected in the optical design, and additional aberrations caused by fabrication and assembly errors. In an FOS, wavefront aberrations at the ROI are corrected by the SLM, with the exception of the RWFE. If the RWFE is very small (diffractionlimited), diffraction becomes the dominant factor affecting the image quality at the ROI. This section covers a derivation of the diffraction MTF of FOSs based on transmissive LC SLMs, including the effect of higher diffraction orders on the MTF contrast.

To simplify calculations, let us assume that the RWFE at the ROI is very small and has no significant effect on the MTF of the FOS at the ROI. Also, if $D \gg a$, the effect of the aperture of diameter $D$ on the MTF is a function that varies very slowly compared to the effect of the SLM amplitude transmission. Therefore, we can first derive the diffraction MTF of the SLM amplitude and then multiply the result by the MTF of the aperture. The SLM amplitude transmission on $x$ can be written as

$p_{S L M}(x)=\operatorname{rect}\left(\frac{x}{b}\right) * \frac{1}{a} \operatorname{comb}\left(\frac{x}{a}\right)$.

The field in the image plane is given by the Fourier transform of the pupil:

$P_{S L M}(\xi)=b \operatorname{sinc}(b \xi) \operatorname{comb}(a \xi)$.

The point spread function (PSF) is the magnitude squared of the field in the image plane, with $\xi=x / \lambda f$ : 


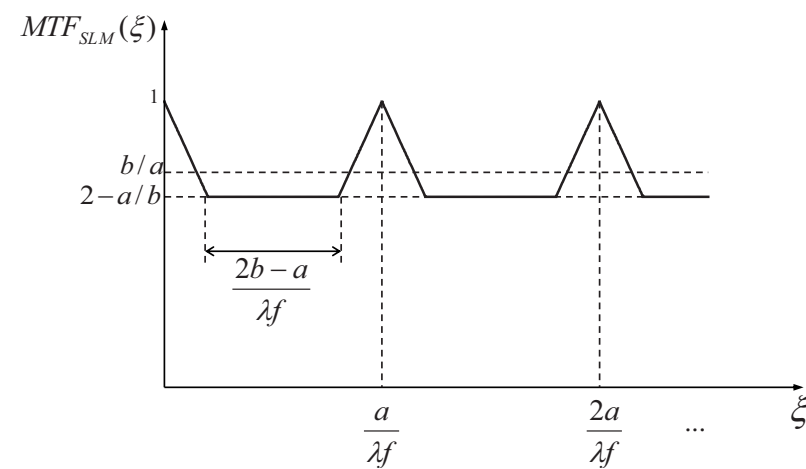

Fig. 7 Diffraction MTF of the SLM amplitude is a sawtooth function with the period $a / \lambda f$ and an average contrast of $b / a$.

$$
\begin{aligned}
\operatorname{PSF}_{S L M}(x) & =|P(\xi)|_{\xi=x / \lambda f}^{2} \\
& =\left(\frac{b}{a}\right)^{2} \operatorname{sinc}^{2}\left(\frac{x}{\lambda f / b}\right) a^{2} \operatorname{comb}^{2}\left(\frac{x}{\lambda f / a}\right) .
\end{aligned}
$$

The optical transfer function (OTF) is the Fourier transform of the PSF, normalized by the total area under the PSF. From the definition of the Fourier transform, the area under the PSF is equal to the Fourier transform of the PSF at $\xi$ $=0$. Since the OTF does not have a phase component in this case, the MTF is equal to the OTF. So the MTF of the SLM amplitude transmission can be written as

$$
\begin{aligned}
\operatorname{MTF}_{S L M}(\xi) & =\left|\frac{\Im\{P S F(x)\}}{\left.\Im\{P S F(x)\}\right|_{\xi=0}}\right| \\
& =\operatorname{tri}\left(\frac{\xi}{b / \lambda f}\right) *\left(\frac{\lambda f}{a}\right)^{2} \operatorname{comb}^{2}\left(\frac{\xi}{a / \lambda f}\right) .
\end{aligned}
$$

The expression in Eq. (16) is a train of triangle functions spaced apart by $a / \lambda f$, forming a sawtooth function with the period $a / \lambda f$ and an average contrast of $b / a$. A schematic plot of this expression is shown in Fig. 7. The flat portion of this sawtooth has a width of $(2 b-a) / \lambda f$ at a contrast of $2-(a / b)$. If the contrast of the flat portion is not greater than 0.5 , the SLM is not suitable for optical foveated imaging, as the MTF would reach a low value periodically, creating artifacts in the image.

The diffraction MTF of the FOS can be calculated by multiplying the MTF of the SLM amplitude by the MTF of the aperture. Assuming that $2-(a / b)>0.5$ and $D \gg a$, we can approximate the high-frequency sawtooth in Eq. (16) by its average value, $b / a$. As a result, the diffraction MTF of an FOS can be written as

$\operatorname{MTF}_{\text {diffr }}(\xi)=\frac{b}{a} \times M T F_{\text {aperture }}(\xi)$.

Equation (17) is valid for any aperture shapes, as long as all the features of the aperture are significantly larger compared to the SLM pixel pitch. If the aperture has any small features comparable in size to the pixel pitch, the diffraction MTF has to be calculated as the convolution between the expression for the MTF of the SLM amplitude from Eq. (16) and the expression for the MTF of the aperture. In our

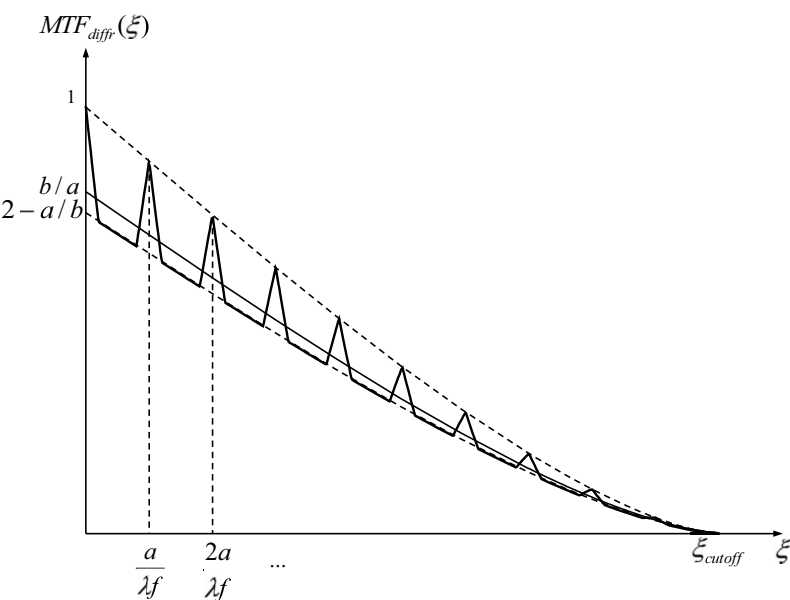

Fig. 8 Diffraction MTF of an FOS with a transmissive LC SLM and a circular aperture.

case, we assumed a circular aperture of diameter $D$, so the MTF of the aperture is given by the following expression:

$$
\begin{aligned}
\operatorname{MTF}_{\text {aperture }}(\xi)= & \frac{2}{\pi}\left\{\cos ^{-1}\left(\frac{\xi}{\xi_{\text {cutoff }}}\right)-\left(\frac{\xi}{\xi_{\text {cutoff }}}\right)\right. \\
& \left.\times\left[1-\left(\frac{\xi}{\xi_{\text {cutoff }}}\right)^{2}\right]^{1 / 2}\right\},
\end{aligned}
$$

where $\xi_{\text {cutoff }}=D / \lambda f$ is the cutoff frequency. ${ }^{24}$ The diffraction MTF of an FOS with a transmissive LC SLM and a circular aperture is schematically plotted in Fig. 8. (The period of the sawtooth is exaggerated for clarity.)

Now, we will quantify the effect of the higher diffraction orders on the image quality. As discussed in the previous section, a portion of the light transmitted through the SLM will end up in higher diffraction orders. Since FOSs image extended objects, the MTF will be further affected by higher orders from other field angles falling onto the image plane. The images formed by the higher diffraction orders will superimpose onto the zero-order image, creating shifted "ghost" images of the extended object, equally spaced on $x$ and $y$. It is very important to estimate the drop in the MTF due to these parasitical images created by the higher diffraction orders.

From Eq. (8), the angular increment between diffraction angles on $x$ and $y, \Delta \beta$, can be approximated by

$\Delta \beta \approx \frac{\lambda}{a}$.

Therefore, the zero-order image at the ROI with the field angle $\left(\theta_{x}, \theta_{y}\right)$ is affected by a superposition of higher diffraction orders, $\left(m_{x}, m_{y}\right)$, each order coming from a different field angle, $\left[\theta_{x}-\left(m_{x} \lambda\right) / a, \theta_{y}-\left(m_{y} \lambda\right) / a\right]$; where $m_{x}, m_{y}$ $=0, \pm 1, \pm 2, \ldots$, with $m_{x}=m_{y} \neq 0$.

In a practical FOS, $a \gg \lambda$, so the angular increment between diffraction angles, $\Delta \beta$, is small relative to the FOV of the FOS. In this case, the wavefront aberration of the optical system and the diffraction efficiency, $\sigma_{m_{x}, m_{y}}$, can be considered constant over a small rectangular FOV centered at the ROI and covering $\pm \Delta \beta$ on $x$ and $y$. Also, in a prac- 


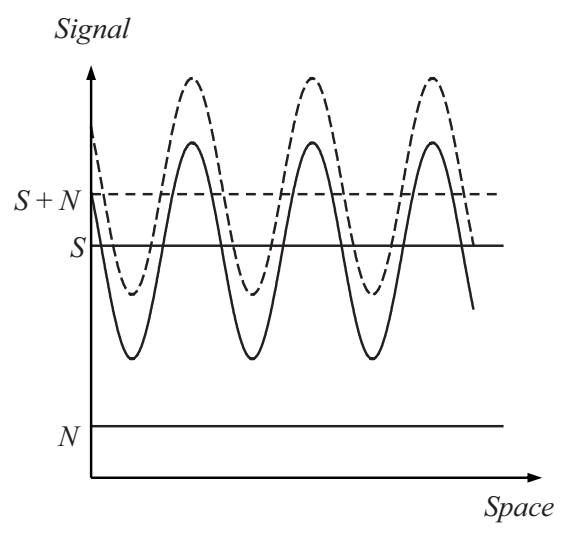

Fig. 9 The effect of higher orders on the zero-order contrast.

tical FOS, most of the transmitted light goes into the zeroorder, and most of the remaining light goes into the eight orders closest to the zero-order. As a result, the total amount of light diffracted from other field angles falling onto the zero-order at the ROI can be approximated by the total diffraction efficiency of the higher orders at the ROI:

$\sigma_{\text {orders }}^{\text {higher }} \approx\left(\frac{b}{a}\right)^{2}-\sigma_{0,0}$,

where $(b / a)^{2}$ is the total transmission of all diffraction orders, and $\sigma_{0,0}$ is the zero-order RMS diffraction efficiency at the ROI, as defined in Eq. (11).

The useful image information is in the zero-order, which can be considered the modulated signal of magnitude $S$. Since the spatial frequencies and contrast variations across an extended object are nondeterministic, the effect of higher diffraction orders from different field angles can be treated as white noise of magnitude $N$, washing down the modulation contrast of the signal $S$. Figure 9 illustrates the effect of higher diffraction orders on the zero-order contrast. The MTF contrast of the zero-order is washed down by a factor equal to $S /(S+N)$. From Eq. (20), $S=\sigma_{0,0}, N$ $=\sigma_{\text {higher orders }}$, and $S+N=(b / a)^{2}$. So the diffraction MTF in Eq. (17) is washed down by a factor equal to

$\frac{S}{S+N}=\frac{\sigma_{0,0}}{(b / a)^{2}}$.

Assuming that the RWFE at the ROI is very small (diffraction-limited), the MTF of the FOS at the ROI can be written as

$\operatorname{MTF}_{R O I}(\xi)=\frac{\sigma_{0,0}}{b / a} \times M T F_{\text {aperture }}(\xi)$.

Figure 10 shows the estimated MTF at the ROI for a diffraction-limited FOS with a circular aperture, an SLM pixel fill factor, $(b / a)^{2}$, and a zero-order diffraction efficiency at the ROI, $\sigma_{0,0}$.

\section{Practical Design Example}

The design proposed by Martinez et al. ${ }^{10}$ illustrates the basic concept of a fast wide-angle FOS using a transmissive SLM. However, although it covers an impressive full FOV

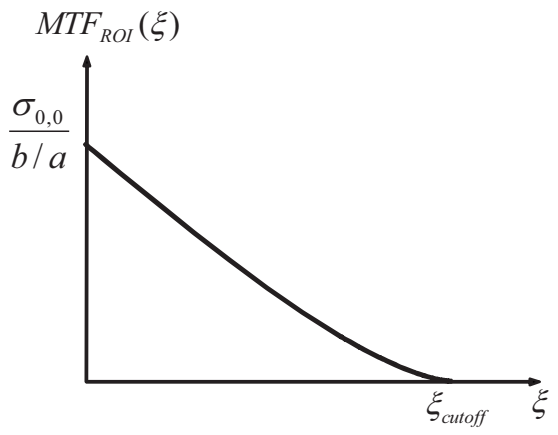

Fig. 10 MTF at the ROI for a diffraction-limited FOS with a circular aperture.

of 90 deg with a very compact two-element optic, the proposed design is not practical because it requires an SLM with a resolution of $2048 \times 2048$ in order to achieve diffraction-limited RWFE. The stop diameter in this design is about $8 \mathrm{~mm}$, so the pixel pitch would have to be $3.9 \mu \mathrm{m}$. Considering a shadow mask width of $2.8 \mu \mathrm{m}$, which is the smallest width achieved so far in transmissive TFT technology, only $8 \%$ of the light would be transmitted, and only about $0.6 \%$ would be in the zero-order. Another disadvantage of this design is that toward the peripheral field angles, the incidence angles to the image plane become very steep. As a result, the relative illumination (RI) at the maximum field angle drops down to almost $20 \%$.

A different wide-angle FOS design was proposed by Harriman et al., ${ }^{20}$ who demonstrated a system covering $120 \mathrm{deg}$, using a transmissive LC SLM with a resolution of $1280 \times 1024$ and a pixel fill factor of $56 \%$. However, this design turned out to be impractical as well, due to the limited zero-order diffraction efficiency and the severe artifacts created by the higher diffraction orders.

In this section, we propose an example of a practical, fast wide-angle FOS design, taking into account the current limitations in the transmissive TFT technology and their effects on the diffraction efficiency and MTF.

\subsection{Optical Design}

The optical design for an 18-mm F/2.8 monochromatic lens has been previously proposed as an example of a fast wideangle lens that can be used in a practical monochromatic FOS based on the current LC SLM technology. ${ }^{25}$ The lens covers a full FOV of $80 \mathrm{deg}$ and matches a sensor with a $25-\mathrm{mm}$ diagonal. The overall length of the optics is $63 \mathrm{~mm}$, with a back focal length of $30 \mathrm{~mm}$. The lens prescription data is given in Table 1, and the optical layout, distortion, and relative illumination plots are shown in Fig. 11. The RMS and P-V wavefront aberrations at $0,10,20,30$, and $40 \mathrm{deg}$ are listed in Table 2. This four-element design is arranged in a retrofocus configuration, with a front negative element followed by a rear positive group. This type of arrangement is typically used in fast wide-angle lenses with aperture ratios ranging from F/4 down to F/1.8 and FOVs larger than 60 deg. ${ }^{26}$

In a wide-angle FOS, the relative illumination (RI) should be flat in order to truly achieve uniform performance at the ROI across the entire FOV. A drop in the RI with the field angle would result in a lower MTF contrast at 
Table 1 Lens prescription data.

\begin{tabular}{|c|c|c|c|c|c|}
\hline $\begin{array}{c}\text { Surface } \\
\#\end{array}$ & Comment & Radius (mm) & $\begin{array}{l}\text { Thickness } \\
(\mathrm{mm})\end{array}$ & Glass & $\begin{array}{l}\text { Diameter } \\
(\mathrm{mm})\end{array}$ \\
\hline 1 & 1st element & 20.199 & 2.700 & BK7 & 23.400 \\
\hline 2 & & 7.734 & 8.100 & Air & 14.400 \\
\hline 3 & $\begin{array}{l}\text { Polarizer } \\
\text { (optional) }\end{array}$ & Infinity & 0.900 & B270 & 14.400 \\
\hline 4 & & Infinity & 0.450 & Air & 14.400 \\
\hline 5 & Filter (optional) & Infinity & 0.900 & F_SILICA & 14.400 \\
\hline 6 & & Infinity & 2.250 & Air & 14.400 \\
\hline 7 & SLM front cover & Infinity & 0.900 & SUPRASIL & 10.800 \\
\hline STOP & SLM back cover & Infinity & 0.900 & SUPRASIL & 9.556 \\
\hline 9 & & Infinity & 4.050 & Air & 10.800 \\
\hline 10 & 2nd element & -26.035 & 3.600 & BK7 & 14.800 \\
\hline 11 & & -13.902 & 0.360 & Air & 18.000 \\
\hline 12 & 3rd element & -194.793 & 3.600 & BK7 & 18.900 \\
\hline 13 & & -25.953 & 0.360 & Air & 21.600 \\
\hline 14 & 4th element & 54.770 & 3.600 & BK7 & 23.400 \\
\hline 15 & & -79.985 & 30.127 & Air & 23.400 \\
\hline 16 & Image plane & Infinity & & & 24.446 \\
\hline
\end{tabular}

the peripheral field angles. In this design, the stop was positioned between the negative and the positive group, as close as possible to the focal point of the positive group, in order to place the exit pupil as far as possible from the image plane. As a result, the RI in this lens is almost flat across the FOV. On the other hand, in wide-angle lenses, it is difficult to flatten the RI and correct the barrel distortion in the same time. However, distortion is only a fielddependent magnification error and does not affect the res-

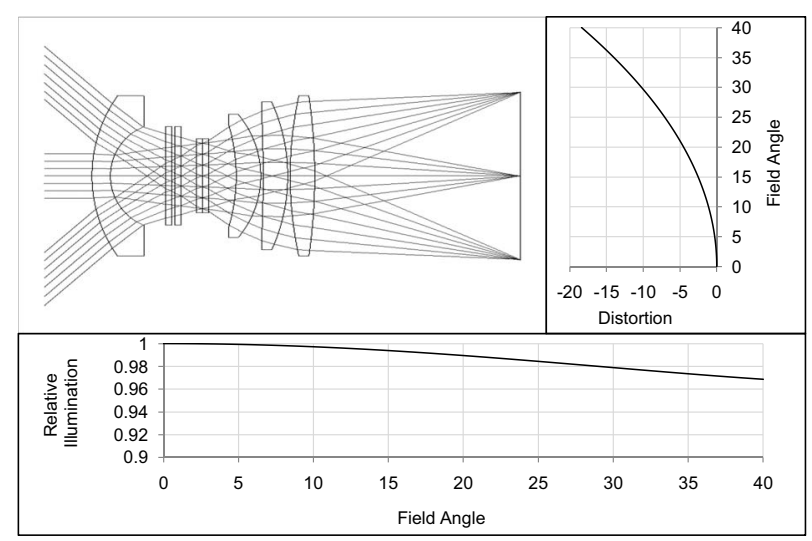

Fig. 11 Optical layout, distortion, and relative illumination. olution of the optics. As long as the resolution of the sensor array is large enough to avoid aliasing due to undersampling, barrel distortion can be calibrated and corrected at the electronics or software level. The barrel distortion in this design is $18 \%$ at the maximum field angle.

Another important aspect in the design of wide-angle FOSs based on transmissive LC SLMs is the size of the stop. Since the SLM is placed at the stop, the pixel pitch is proportional to the stop diameter. So for a given SLM resolution and shadow mask width, a larger stop will result in a larger fill factor. In this design, the stop was positioned as far as possible behind the negative front element in order to increase as much as possible the stop diameter relative to the entrance pupil diameter. The stop in this lens is $50 \%$ larger than the entrance pupil.

Table 2 RMS and P-V wavefront aberrations of the uncorrected lens.

\begin{tabular}{lccccc}
\hline \hline Field angle in degrees & 0 deg & $10 \mathrm{deg}$ & $20 \mathrm{deg}$ & $30 \mathrm{deg}$ & $40 \mathrm{deg}$ \\
\hline RMS WFE in waves & 0.74 & 0.69 & 0.67 & 0.87 & 1.28 \\
P-V WFE in waves & 2.95 & 2.71 & 3.29 & 4.98 & 9.01 \\
\hline \hline
\end{tabular}


Table 3 Performance at the ROI with five different SLM resolutions (ROI at $40 \mathrm{deg}$ ).

\begin{tabular}{lccccc}
\hline \hline SLM resolution & $32 \times 32$ & $64 \times 64$ & $128 \times 128$ & $256 \times 256$ & $512 \times 512$ \\
\hline Pixel pitch in $\mu \mathrm{m}$ & 300 & 150 & 75 & 37 & 19 \\
Fill factor, $(b / a)^{2}$ & 0.98 & 0.96 & 0.93 & 0.85 & 0.73 \\
Zero-order efficiency, $\sigma_{0,0}$ & 0.68 & 0.79 & 0.81 & 0.72 & 0.52 \\
MTF at $\xi=0$ & 0.69 & 0.80 & 0.85 & 0.78 & 0.61 \\
\hline \hline
\end{tabular}

\subsection{Optimal SLM Resolution}

The resolution of the transmissive LC SLM has to be carefully selected in order to optimize the FOS performance. If the SLM resolution is too low, the RWFE will affect the diffraction efficiency and MTF of the system. On the other hand, since the minimum shadow mask width is limited by the current TFT technology, increasing the SLM resolution for a given aperture size will decrease the fill factor, also affecting the diffraction efficiency and MTF. Therefore, choosing the optimal SLM resolution is a trade-off between minimizing the amplitude diffraction effects caused by the shadow mask and minimizing the phase diffraction effects caused by the RWFE.

In this design, the SLM resolution was optimized based on the largest wavefront aberration, which occurs at the 40-deg field angle. The 2-D wavefront aberration map at the 40-deg field angle was obtained directly from the raytracing program used for this lens design. The values for the local P-V RWFE on $x$ and $y$ at each pixel $[i, j]$, $R W F E_{P-V x, y}[i, j]$, as defined in Eq. (3), were determined from the wavefront map, for five different SLM resolution scenarios: $32 \times 32,64 \times 64,128 \times 128,256 \times 256$, and $512 \times 512$. For an SLM resolution of $N \times N$, the ratio $b / a$ can be calculated as

$\frac{b}{a}=1-\frac{w}{a}=1-\frac{N w}{D_{\text {Stop }}}$,

where $w$ is the width of the shadow mask, and $D_{\text {Stop }}$ is the pupil stop diameter. In this design, $D_{S t o p}=9.556 \mathrm{~mm}$, and

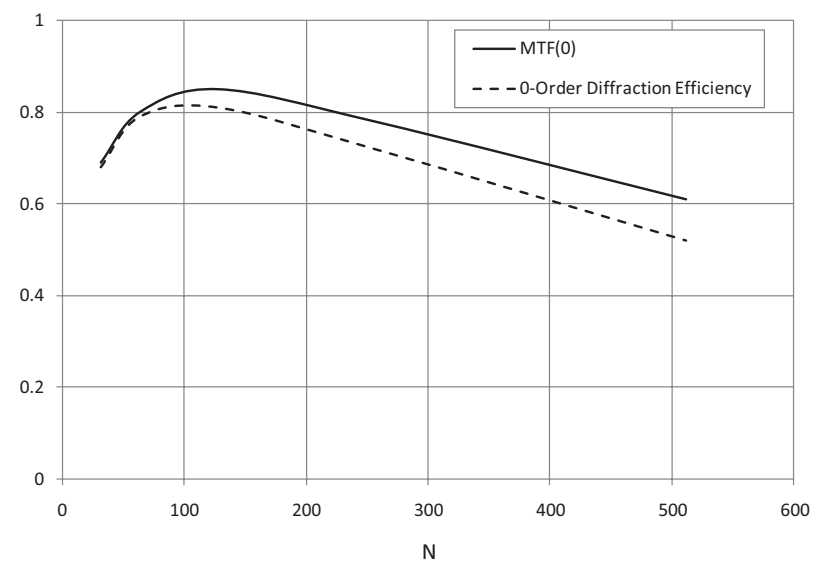

Fig. $12 \sigma_{0,0}$ and MTF at $\xi=0$ versus $N$ (ROI at $40 \mathrm{deg}$ ). we considered $w=2.8 \mu \mathrm{m}$, which is the smallest shadow mask width achieved so far in the transmissive TFT technology. The $b / a$ values obtained from Eq. (23) and the $R W F E_{P-V x, y}[i, j]$ values obtained from the wavefront map were used in Eqs. (10) and (11) to calculate the zero-order diffraction efficiency, $\sigma_{0,0}$. The MTF at $\xi=0$ was calculated from Eq. (22) as $\sigma_{0,0} /(b / a)$. Table 3 lists the pixel pitch, fill-factor, $\sigma_{0,0}$, and $\operatorname{MTF}(\xi=0)$, for the five different SLM resolutions considered, with the ROI at $40 \mathrm{deg}$.

Figure 12 shows plots of $\sigma_{0,0}$ and $\operatorname{MTF}(\xi=0)$ versus $N$, where $N \times N$ is the SLM resolution. The optimal SLM resolution for this FOS design example is $128 \times 128$. For lower SLM resolutions, the phase diffraction caused by the RWFE is the main factor limiting the diffraction efficiency and the image quality at the ROI. For higher SLM resolutions, the amplitude diffraction caused by the shadow mask becomes the dominant factor affecting the performance of the FOS at the ROI.

The zero-order diffraction efficiency and $\operatorname{MTF}(\xi=0)$ at the ROI were also calculated with the correction applied at 0 , 10, 20, and $30 \mathrm{deg}$, for an SLM resolution of 128 $\times 128$ (listed in Table 4). Notice that the performance at the $\mathrm{ROI}$ is almost uniform across the entire FOV, a result of the wavefront aberration being distributed somewhat evenly over the FOV.

Now what happens at the other field angles when the wavefront aberration is corrected at the ROI? In addition to the amplitude and phase diffraction effects caused by the pixelated structure of the SLM, these field angles are also affected by the uncorrected wavefront aberration, $W$ $-W_{R O I}$, where $W$ is the initial aberration, and $W_{R O I}$ is the wavefront correction applied at the ROI. Table 5 lists the zero-order diffraction efficiency, $\operatorname{MTF}(\xi=0)$, and the wavefront aberration at different field angles when correction is applied at $40 \mathrm{deg}$. Notice that even $5 \mathrm{deg}$ away from the ROI, at the 35-deg field angle, $\sigma_{0,0}$ and $\operatorname{MTF}(\xi=0)$ do not change significantly. However, aberrations in fast wide-

Table 4 Performance at the ROI with correction at different field angles $(128 \times 128$ SLM resolution)

\begin{tabular}{lccccc}
\hline \hline Field angle in degrees & 0 deg & $10 \mathrm{deg}$ & $20 \mathrm{deg}$ & $30 \mathrm{deg}$ & $40 \mathrm{deg}$ \\
\hline Zero-order efficiency, $\sigma_{0,0}$ & 0.83 & 0.83 & 0.83 & 0.82 & 0.81 \\
MTF at $\xi=0$ & 0.86 & 0.86 & 0.86 & 0.85 & 0.85 \\
\hline \hline
\end{tabular}


Table 5 Performance with correction applied at 40 deg $(128 \times 128$ SLM resolution).

\begin{tabular}{lccccc}
\hline \hline Field angle in degrees & $40 \mathrm{deg}$ & $39 \mathrm{deg}$ & $35 \mathrm{deg}$ & $0 \mathrm{deg}$ & $-40 \mathrm{deg}$ \\
\hline Zero-order efficiency, $\sigma_{0,0}$ & 0.81 & 0.81 & 0.80 & 0.78 & 0.70 \\
MTF at $\xi=0$ & 0.85 & 0.85 & 0.84 & 0.81 & 0.74 \\
RMS aberration in waves & 0 & 0.09 & 0.39 & 1.40 & 2.43 \\
P-V aberration in waves & 0 & 0.56 & 2.50 & 8.91 & 17.8 \\
\hline \hline
\end{tabular}

angle lenses change rapidly with the field angle. As a result, aberrations become the dominant factor affecting the image quality away from the ROI. Figure 13 shows the estimated MTF of the FOS with the wavefront aberration corrected at $40 \mathrm{deg}$.

\section{Summary}

This paper proposed an approach to the analysis of fast wide-angle monochromatic FOSs based on the current transmissive LC SLM technology. The effect of the SLM resolution and pixel fill factor on the diffraction efficiency and image quality of these systems was quantified. The design example presented here reveals additional challenges specific to the optical design of fast wide-angle FOSs, such as controlling the RI, distortion, and aberrations across a wide FOV.

The shadow mask and the discrete piston-only OPD are the main factors affecting the zero-order diffraction efficiency and image quality at the ROI in FOSs using transmissive LC SLMs. Choosing the optimal SLM resolution for a given lens design is a trade-off between minimizing the amplitude diffraction effects caused by the shadow mask and minimizing the phase diffraction effects caused by the discrete piston-only correction.

The theoretical study presented in this paper sets basic design, optimization, and analysis guidelines for future developments in fast wide-angle FOSs based on transmissive SLM devices.

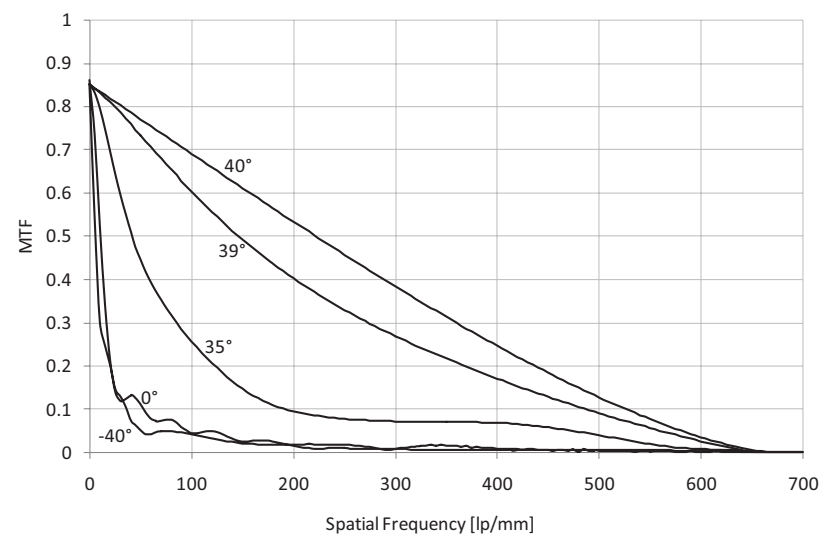

Fig. 13 MTF of the FOS with the aberrations corrected at 40 deg.

\section{References}

1. N. Tsumura, C. Endo, H. Haneishi, and Y. Miyake, "Image compression and decompression based on gazing area," Proc. SPIE $\mathbf{2 6 5 7}$ 361-367 (1996).

2. P. Kortum and W. Geisler, "Implementation of a foveated image coding system for image bandwidth reduction," Proc. SPIE 2657, 350 360 (1996).

3. W. S. Geisler and J. S. Perry, "A real-time foveated multi-resolution system for low-bandwidth video communication," Proc. SPIE 3299, 294-305 (1998).

4. A. Ude, C. Gaskett, and G. Cheng, "Foveated vision systems with two cameras per eye," in Proc. IEEE Int. Conf. Robotics and Automation, Orlando, Florida, pp. 3457-3462 (2006).

5. H. Hua and S. Lin, "Dual-sensor foveated imaging system," Appl. Opt. 47(3), 317-327 (2008).

6. Z. Zhou, B. Pain, and E. R. Fossum, "Frame-transfer CMOS active pixel sensor with pixel binning," IEEE Trans. Electron Devices 44(10), 1764-1768 (1997).

7. J. Coulombe, M. Swan, and C. Wang, "Variable resolution CMOS current mode active pixel sensor," in Proc. IEEE Int. Symp. Circuits and Systems (ISCAS 2000), vol. 2, pp. 293-296 (2000).

8. F. Saffih and R. Hornsey, "Multiresolution CMOS image sensor," in Tech. Digest SPIE, Opto-Canada 2002, p. 425-428, SPIE Press, Belligham, WA (2002).

9. K. Wakamiya, T. Senga, K. Isagi, N. Yamamura, Y. Ushio, and N. Kita, "A new foveated wide angle lens with high resolving power and without brightness loss in the periphery," Proc. SPIE 6051, 605107 (2005)

10. T. Martinez, D. V. Wick, and S. R. Restaino, "Foveated, wide fieldof-view imaging system using a liquid crystal spatial light modulator," Opt. Express 8(10), 555-560 (2001).

11. D. V. Wick, D. M. Payne, T. Martinez, and S. R. Restaino, "Large dynamic range wavefront control of micromachined deformable membrane mirrors," Proc. SPIE 5798, 158-161 (2005).

12. J. Harriman, S. Serati, and J. Stockley, "Comparison of transmissive and reflective spatial light modulators for optical manipulation applications," Proc. SPIE 5930, 59302D (2005).

13. M. T. Gruneisen, R. C. Dymale, J. R. Rotgé, D. G. Voelz, and Melinda Deramo, "Wavelength-agile telescope system with diffractive wavefront control and acousto-optic spectral filter," Opt. Eng. 44(10), 103202 (2005).

14. B. E. Bagwell, D. V. Wick, W. D. Cowan, O. B. Spahn, W. C. Sweatt, T. Martinez, S. R. Restaino, J. R. Andrews, C. C. Wilcox, D. M. Payne, and R. Romeo, "Active zoom imaging for operationally responsive space," Proc. SPIE 6467, 64670D (2007).

15. X. Zhao, "Broadband and wide field of view foveated imaging system in space," Opt. Eng. 47(10), 103202 (2008).

16. D. V. Wick, T. Martinez, S. R. Restaino, and B. R. Stone, "Foveated imaging demonstration," Opt. Express 10(1), 60-65 (2002).

17. G. Curatu, D. V. Wick, D. M. Payne, T. Martinez, J. Harriman, and J. Harvey, "Wide field-of-view imaging system using a liquid crystal spatial light modulator," Proc. SPIE 5874, 587408-1-7 (2005).

18. X. Zhao, Y. Xie, and W. Zhao, "Wide field-of-view foveated imaging system," Chin. Opt. Lett. 6(8), 561-563 (2008).

19. G. D. Love, "Wave-front correction and production of Zernike modes with a liquid-crystal spatial light modulator," Appl. Opt. 36(7), 15171524 (1997).

20. J. Harriman, S. Gauza, S.-T. Wu, D V Wick, B. Bagwell, T Martinez, D. M. Payne, and S. Serati, "Transmissive spatial light modulators with high figure-of-merit liquid crystals for foveated imaging applications," Proc. SPIE 6135, 61350C (2006).

21. B. E. Bagwell, D. V. Wick, and J. Schwiegerling, "Multi-spectral foveated imaging system," in Proc. IEEE Aerospace Conference 2006, pp. 1114-1121, IEEE, Piscataway, NJ (2006). 
22. B. E. Bagwell, D. V. Wick, R. Batchko, J. D. Mansell, T. Martinez, S. R. Restaino, D. M. Payne, J. Harriman, S. Serati, G. Sharp, and J. Schwiegerling, "Liquid crystal based active optics," Proc. SPIE 6289, $628908(2006)$.

23. J. W. Goodman, Fourier Optics, pp. 104-105, Roberts \& Company, Englewood, CO (2005).

24. G. D. Boreman, Modulation Transfer Function in Optical and Electro-Optical Systems, p. 22, SPIE Press, Bellingham, WA (2001).

25. G. Curatu and J. E. Harvey, "Lens design and system optimization for foveated imaging," Proc. SPIE 7060, 70600P (2008)

26. W. J. Smith, Modern Lens Design, pp. 147-167, McGraw-Hill, New York (1992)

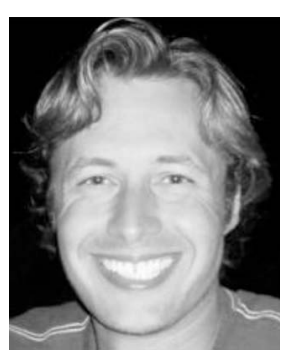

George Curatu obtained his bachelor's degree in electrical engineering from Ryerson Polytechnic University in 1999, his MS in electrical engineering from Laval University in 2000, and his MS in optics from the University of Central Florida in 2004. From 2001 to 2003, he was a senior optical engineer with Spectra-Physics. Since 2005, he has been the optical engineering manager at LightPath Technologies. Mr. Curatu is also continuing his studies toward a PhD in optics at the University of Central Florida, where his research interests include optical design, testing, and image analysis. He is a member of SPIE.

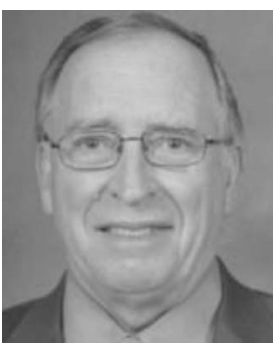

James E. Harvey is an associate professor of optics and senior research staff member at the Center for Research and Education in Optics and Lasers (CREOL) at the University of Central Florida. His formal training included an $A B$ degree in physics from Kansas Wesleyan University and an MS degree in physics from Wayne State University. Since receiving his $\mathrm{PhD}$ in optical sciences from the University of Arizona in 1976, Dr. Harvey has been extensively involved in various aspects of surface scatter phenomena and $\mathrm{x}$-ray imaging systems. Dr. Harvey is credited with three patents and over one hundred publications and presentations in the areas of diffraction theory, surface scatter phenomena, image analysis, x-ray/EUV imaging systems, phased telescope arrays, adaptive optics, wavefront sensing, beam sampling technology, and optical properties of materials. He is a fellow of SPIE and a member of the OSA. 